\title{
Umbilical fibrous hamartoma of infancy: a case report
}

\section{Hamartoma fibroso de la infancia umbilical: reporte de un caso}

\author{
Paula Muñoz $^{\mathrm{a}, \mathrm{b}}$, Roberto Bustos $^{\mathrm{b}}$, Jocelyn Manfredi ${ }^{\mathrm{c}}$ \\ ${ }^{a}$ Dermatology unit, Hospital Luis Calvo Mackenna, Santiago, Chile \\ bDepartament of Dermatology, Faculty of Medicine, University of Chile, Santiago, Chile \\ cPathological Anatomy Unit, Clínica Santa María, Santiago, Chile
}

Received: 7-5-2018; Approved: 15-7-2018

\section{Keywords:}

Fibrous hamartoma of infancy; congenital; umbilical; soft tissue tumour 


\section{Introduction}

Soft tissue tumors in children are diverse and often complex diagnostic entities. Therefore, an exhaustive clinical evaluation with a complete histological study is essential to differentiate the diverse entities and thus to establish an accurate treatment and prognosis.

Fibrous hamartoma of infancy (FHI) is an uncommon benign fibroproliferative tumor with a characteristic three-phase morphology ${ }^{1}$. It was first described by Reye in 1956 as a subdermal fibrous tumor of infancy ${ }^{2}$ and was renamed by Enzinger in 1965 with the current name ${ }^{3}$. The WHO classification of soft tissue tumors defines it as a fibroblastic/myofibroblastic tumor poorly defined, with a characteristic histologic pattern ${ }^{1}$.

It usually occurs before the age of two as a single, asymptomatic lesion located in the subcutaneous cellular tissue or in the reticular dermis, and it is difficult to differentiate from adjacent healthy tissue ${ }^{4,5}$. Due to its similarity with several entities, its diagnosis must be confirmed by a histological and immunohistochemical study. However, these methods may have limitations since their findings are sometimes unspecific.

Although nearly 200 cases have been published in the international literature to date, there are few reports in the Latin American population. The objective of this study is to describe a case of congenital FHI in an infant, with atypical clinical and histological characteristics, where additional molecular studies were needed to differentiate it from other skin conditions.

\section{Clinical case}

Full-term male newborn, controlled pregnancy, eutocic delivery, with no perinatal morbid history. He was referred to the dermatology department due to a slightly infiltrated erythematous plaque in the peri and infra-umbilical region which was present since birth, with well-defined borders and adherent superficial yellowish peeling at the lower pole, without other local inflammatory signs (Figure 1). A skin ultrasound study revealed homogeneous hypoechoic dermoepidermal lesion, with slight extension to the subcutaneous tissue and few sinuous vessels in its deepest segment.

Given the clinical characteristics, a deep incisional biopsy was performed which showed proliferation of thin fusiform cells in dermis and hypodermis, of biphasic morphology with one segment arranged in a infiltrate swirling pattern and another one with fused cell bands with fibroblastic and myofibroblastic ap- pearance, and with absence of Grenz zone (Figure $2 \mathrm{~A}$ and $\mathrm{B})$. It presented in depth a component of mature adipose tissue with no signs of atypia (Figure 3 ). Mature epidermis, partially atrophic. There was no atypical necrosis or mitosis. The immunohistochemical study of the tumor cells showed intense and diffuse positivity for CD34 (Figure 4) and focal FXIIIa positivity, with the absence of immunoreactivity for actin, desmin, MyoD1, S100, HMB45, MelanA, and EMA. Moderate Ki-67 proliferation index, with positivity in approximately $20 \%$ of cells. The study was complemented with FISH using PDGF beta and ETV6, which were negative, excluding differential diagnoses of congenital dermatofibrosarcoma protuberans and infantile fibrosarcoma, respectively. These antecedents, along with the histological findings, were consistent with the diagnosis of FHI.

Surgical extirpation was performed by the pediatric surgery team, with umbilical readjustment through lateral rotation flaps. The histological and immunohistochemical study supported the previously described findings, compromising margins of surgical resection. The patient evolved without signs of recurrence at seven months of follow-up (Figure 5).

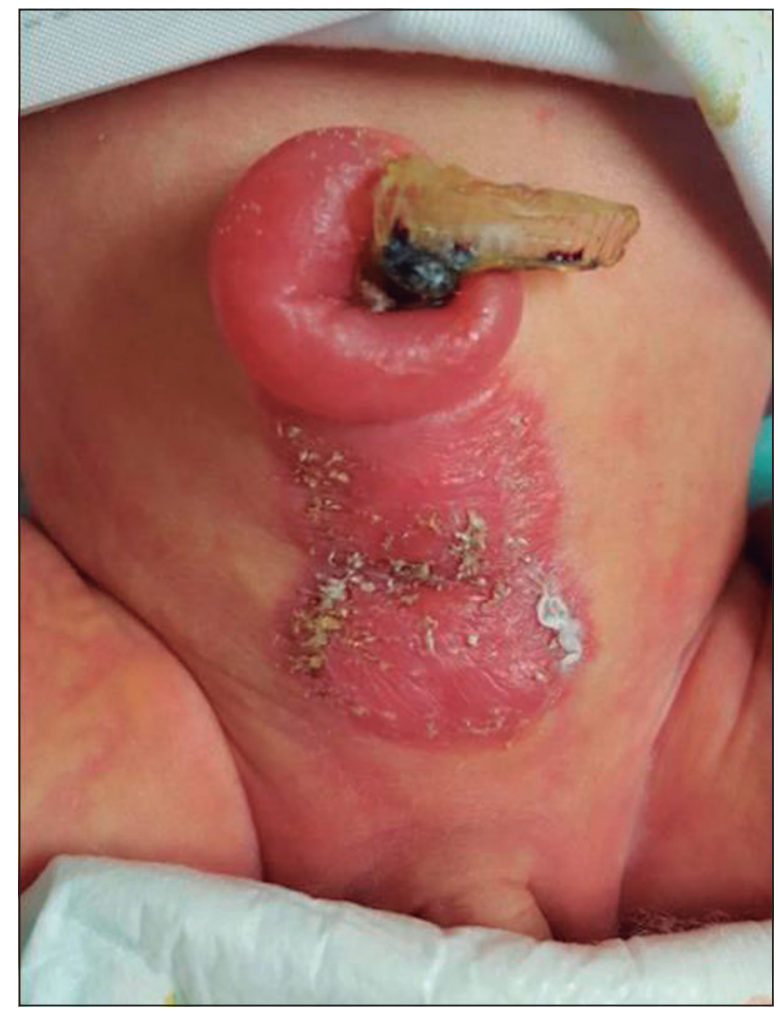

Figure 1. Fibrous hamartoma of infancy: erythematous plaque slightly infiltrated in the peri and infraumbilical region. 


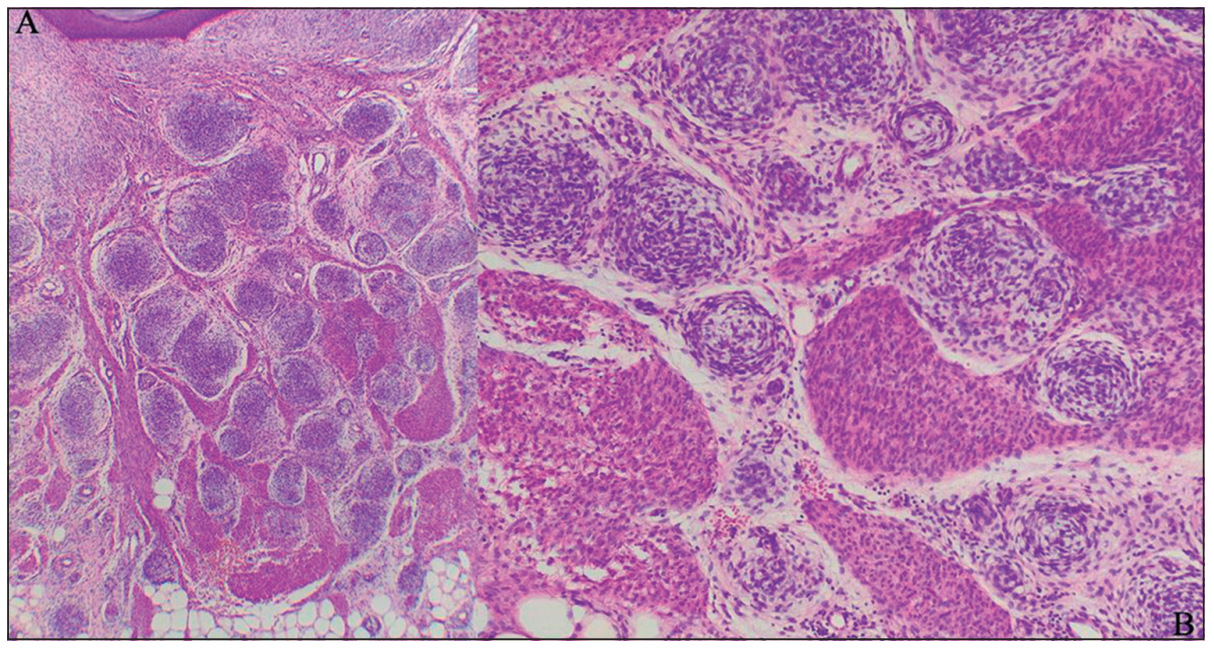

Figure 2. Optical microscopy. A, with $2 x$ hematoxylin-eosin staining, skin with mature, focally atrophic epidermis, associated with a spindle fusocellular lesion, with biphasic embryonic stroma. In B, with 20x hematoxylin-eosin staining, spindle cell injury with embryonic stroma was observed, forming swirling structures and bands of fused cells. No necrosis or atypical mitosis is observed.

\section{Discussion}

FHI most frequently affect males ${ }^{4,5}$, generally occurring during the first two years of life, with an average age ranging from 15 and 18 months $s^{4,5}$. Although congenital lesions have been described as in our case, they represent between $4-23 \%$ of the total according to various reports ${ }^{4,6}$. It does not show family history and it is not related to other neoplasms or congenital disorders 5 .

Currently, there are controversies regarding the origin of this entity. Cytogenetic abnormalities involving different chromosomes have recently been identified $^{7-9}$, supporting the theory that FHI is a neoplastic rather than a hamartomatous process.

Classically, it is presented as a single, mobile, small, poorly limited, asymptomatic, skin-colored, firm nodule with no associated epidermal changes. It is located mainly in armpits, back, and upper extremities ${ }^{4,5}$. Although most patients share the described characteristics, multiple lesions ${ }^{6}$ and changes in adjacent skin have been reported, such as alteration in pigmentation $^{10}$, hyperplasia of eccrine glands ${ }^{11}$, and hypertricho$\operatorname{sis}^{12}$. It is interesting in our case the observed epidermal changes, as well as the anatomical location. Generally, the FHI increases in size up to five years and then slows its growth, but without stopping or regressing ${ }^{13}$. On the other hand, there are reports of accelerated growth during childhood ${ }^{13}$. However, it is important to note that in most of the published series a smaller percentage of patients have completed clinical follow-up.

Histologically, the tumor may be subcutaneous or adhered to the dermis. It is characterized by a variable three-phase component of mature fibroblastic-myofibroblastic tissues, immature mesenchymal and mature adipose tissues, frequently associated with chronic inflammatory infiltrate ${ }^{5}$. These components vary in proportion but maintain a uniform general morphology. It

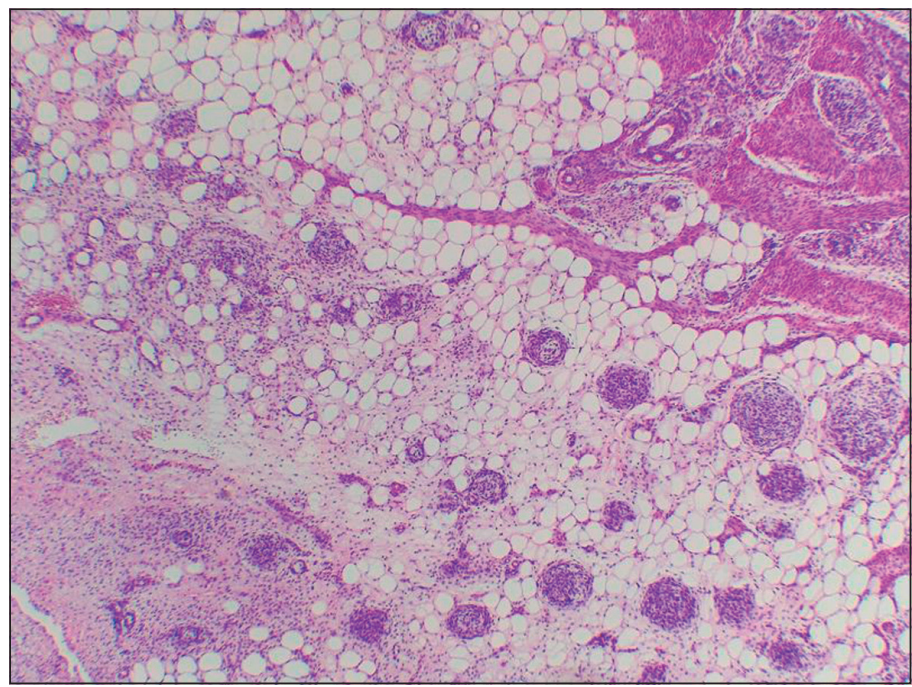

Figure 3. Optical microscopy. Hematoxylin-eosin stain 4x. Fusocellular lesion associated with mature adipose tissue.

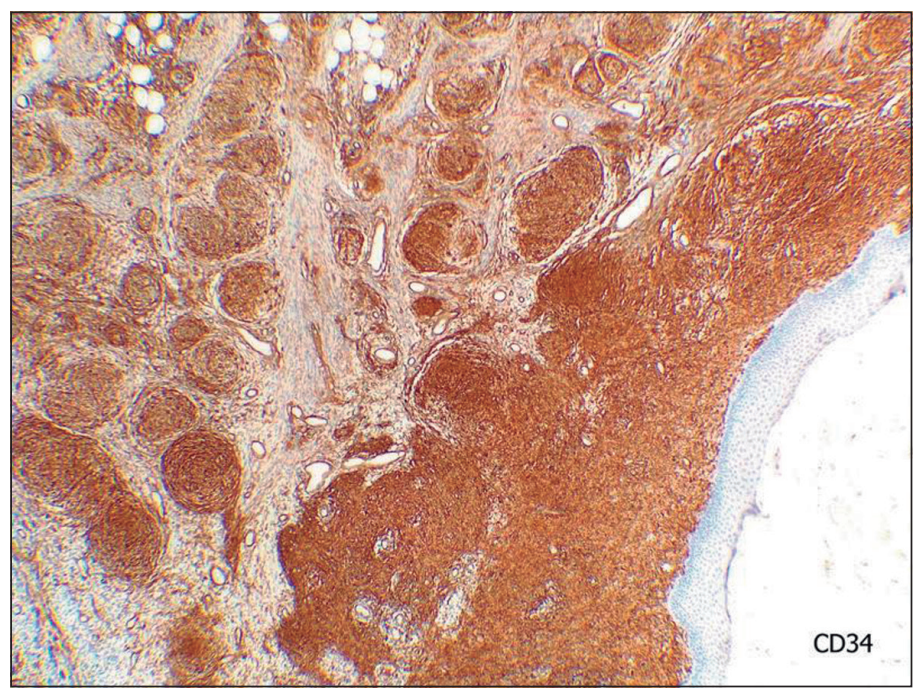

Figure 4. Optical microscopy. Immunohistochemical study CD34. Positivity is observed in the fused cells, with membrane and cytoplasm pattern. 
Figure 5. Fibrous hamartoma of infancy: peri and infraumbilical scar (7 months post surgery).

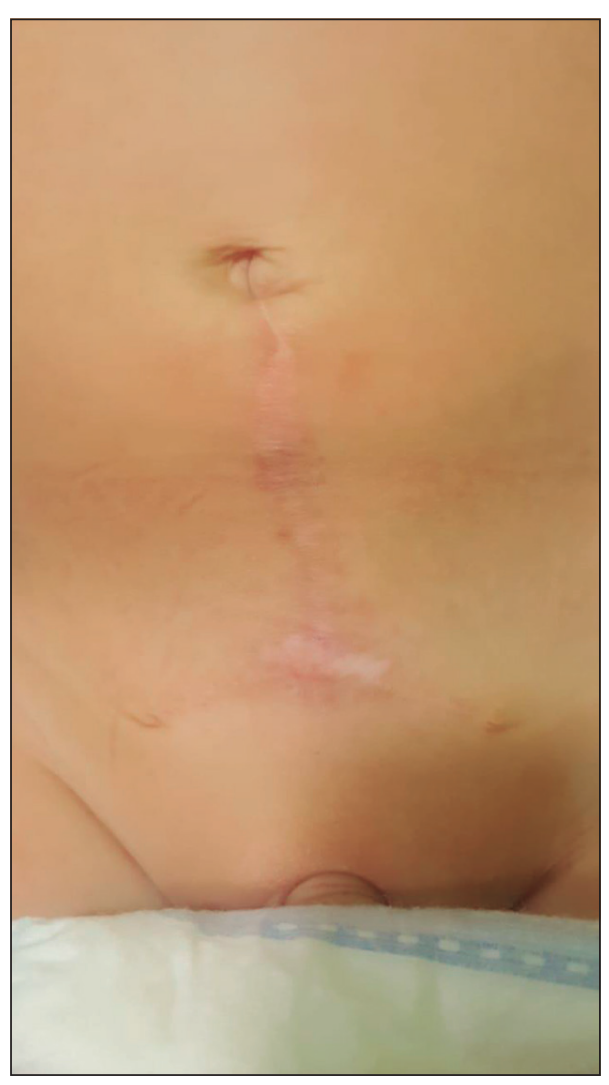

allows the evaluation of chromosomes through fluorescence emission. In our case, given the histological similarity with congenital dermatofibrosarcoma protuberans, FISH was requested for PDGF beta-receptor, which reinforced the diagnosis of FHI, as well as in the literature ${ }^{5}$. In addition, the study was complemented with FISH for ETV6 present in infantile fibrosarcoma, which was also negative.

The use of nuclear magnetic resonance as a complementary diagnostic element has recently been reported, showing an organized fat-like structure with heterogeneous soft tissue interleave bands ${ }^{16}$.

The differential diagnosis depends on the dominant tissue component, which includes benign tumors such as lipomas, neurofibromas, dermatofibromas, lymphadenopathies, and vascular malformations, as well as malignant tumors such as lymphomas or sarcomas.

The treatment of choice is a surgical removal, although sometimes it is not possible due to cosmetic and/or functional implications. A recurrence rate of approximately $15 \%$ has been reported secondary to incomplete resections ${ }^{6}$. Although it does not remit spontaneously, there are no cases of malignancy described ${ }^{4}$.

\section{Conclusions}

As physicians, it is important to consider FHI within the differential clinical and histological diagnosis of childhood subcutaneous tumors, especially in patients under two years of age and those located in the armpit, the back, and the upper extremities.

Although its behavior is benign, its location, size, and similarity with malignant lesions generate anxiety in the family group, which makes it necessary to maintain a good therapeutic link, always highlighting the need for histological, immunohistochemical, and molecular biological studies to differentiate it from other entities.

Despite the fact that surgical extirpation is the treatment of choice, we must consider the functional and/or cosmetic implications involved, which may require a therapeutic approach along with surgeons, pediatricians, and dermatopathologists, which was our case.

\section{Ethical Responsibilities}

Human Beings and animals protection: Disclosure the authors state that the procedures were followed according to the Declaration of Helsinki and the World Medical Association regarding human experimentation developed for the medical community. 
Data confidentiality: The authors state that they have followed the protocols of their Center and Local regulations on the publication of patient data.

Rights to privacy and informed consent: The authors have obtained the informed consent of the patients and/or subjects referred to in the article. This document is in the possession of the correspondence author.

\section{Financial Disclosure}

Authors state that no economic support has been associated with the present study.

\section{Conflicts of Interest}

Authors declare no conflict of interest regarding the present study.

\section{References}

1. Coffin CM. Fibrous hamartoma of infancy. In: Fletcher CDM, Bridge JA, Hogendoorn PCW, Mertens F, eds. WHO Classification of Tumours of Soft Tissue and Bone. Lyon, France: IARC; 2013: 54-55.

2. Reye RD. A consideration of certain subdermal fibromatous tumours of infancy. J Pathol Bacteriol 1956; 72:149e54.

3. Enzinger FM. Fibrous hamartoma of infancy. Cancer 1965; 18:241-8.

4. Al-Ibraheemi A, Martinez A, Weiss SW, Kozakewich HP, Perez-Atayde AR, Tran H, Parham DM, Sukov WR, Fritchie KJ, Folpe AL. Fibrous hamartoma of infancy: a clinicopathologic study of 145 cases, including 2 with sarcomatous features. Mod Pathol 2017; 30:474-485.

5. Saab ST, McClain CM, Coffin CM. Fibrous hamartoma of infancy: a clinicopathologic analysis of 60 cases. Am
J Surg Pathol. 2014; 38:394-401.

6. Dickey GE, Sotelo-Avila C. Fibrous hamartoma of infancy: current review. Pediatr Dev Pathol. 1999; 2:236-243.

7. Lakshminarayanan R, Konia T, Welborn J. Fibrous hamartoma of infancy: a case report with associated cytogenetic findings. Arch Pathol Lab Med. 2005; 129:520-522.

8. Rougemont AL, Fetni R, Murthy S, et al. A complex translocation $(6 ; 12 ; 8)$ ( $225 ; \mathrm{q} 24.3 ; \mathrm{q} 13)$ in a fibrous hamartoma of infancy. Cancer Genet Cytogenet. 2006; 171:115-118.

9. Tassano E, Nozza P, Tavella E, et al. Cytogenetic characterization of a fibrous hamartoma of infancy with complex translocations. Cancer Genet Cytogenet. 2010; 201:66-69.

10. Sotelo-Avila C, Bale PM. Subdermal fibrous hamartoma of infancy: pathology of 40 cases and differential diagnosis. Pediatr Pathol. 1994; 14:39-52.

11. Grynspan D, Meir K, Senger C, et al.
Cutaneous changes in fibrous hamartoma of infancy. J Cutan Pathol 2007; 34:39-43.

12. Yoon TY, Kim JW. Fibrous hamartoma of infancy manifesting as multiple nodules with hypertrichosis. J Dermat 2006; 33:427-9.

13. Efem SEE, Ekpo MD. Clinicopathological features of untreated fibrous hamartoma of infancy. J Clin Pathol. 1993; 46:522524.

14. Coffin CM, Alaggio R. Fibroblastic and myofibroblastic tumors in children and adolescents. Pediatr Dev Pathol. 2012; 15:127-180.

15. Yu G, Wang Y, Wang G, Zhang D, Sun Y. Fibrous hamartoma of infancy: a clinical pathological analysis of seventeen cases. Int J of Clin Exp Pathol. 2015; 8(3):33743377.

16. Stensby JD, Conces MR, Nacey NC. Benign fibrous hamartoma of infancy: a case of MR imaging paralleling histologic findings. Skeletal Radiol 2014;43: 16391643. 